\title{
XXVII.
}

Aus der medicinisohen Klinik zu Breslau.

\section{Das Verhalten von Adenin und Guanin im thierischen Organismus. Von}

Dr. Alfred Schittenhelm, Assistenzarzt.

Minkowski1) hat dadureh, dass es ihm gelang, bei Verfutterung freien Hypoxanthins an Mensehen und Hunden eine gesteigerte Ausscheidung von Harnsäure mit dem Harne nachzuweisen, den directen Uebergang der genannten Base in Harnsäure bewiesen. Dadurch hat er die vor dieser bemerkenswerthen Entdeckung allgemein gultige Ansicht, dass nur Nucleinsubstanzen, in welchen die Purinbasen (also auch Hypoxanthin) in hydrolytisch abspaltbarer Form sich befinden, das Material für die Bildung der Harnsäure im thierischen Organismus liefern, endgtiltig widerlegt.

Dem Hypoxanthin ist seiner Constitution nach das A de nin nahe verwandt. Es geht nicht nur durch chemische Agentien, wie $\mathrm{HNO}_{2}$, sondern nach Schindler ${ }^{2}$ auch durch Fäulniss und vermuthlich auch im thierisehen Organismus in Hypoxanthin tiber.

Versuche, welche Minkowski mit diesem Körper an Hunden anstellte, liessen daher von vornherein ein ähnliches Resultat, wie bei Verfutterung von Hypozanthin, erwarten. Sie verliefen jedoch in durehaus anderer Richtung: Es fand weder eine Vermehrung der Harnsăure statt, noch war Allantoin nachzuweisen (welches doch aus Hypoxanthin zu 70 Proc. entsteht). Dagegen fanden sich schwere anatomische Veränderungen in der Niere der Versuchsthiere, welche dureh Ablagerung von krystallinischer Harnsäure und von harnsaurem Ammonium hervorgerufen wurden.

1) Minkowski, Untersuchungen zur Physiologie u. Pathologie der Harnsäure b. Säugethieren. Arch. f. experim. Pathol. u. Pharmakol., Bd. XLI, S. 375 f.

2) Schindler, Beitr. zur Kenntniss des Adenin u. Guanin u. ihrer Derivate. Zeitschr. f. physiol. Chemie, Bd. 13, S. 432. 
Ferner wurde bei Minkowski's Versuchen mit Adenin beobachtet, dass das letztere auf den Organismus des $\mathrm{H}$ undes eine stark giftige Wirkung zeigte, sich äussernd in intensiven Krankheitserscheinungen, die mehrfach mit dem Tode des Thieres endeten. Abgesehen von gewissen Erscheinungen von Seiten des Circulationsapparates traten heftige Störungen im Intestinaltractus auf, welche ihren anatomischen Grund hatten in intensiver Entzündung der Darmschleimhaut, in geringerem Grade auch der Magenschleimhaut.

Da sich nach diesen Versuchen am Hunde das Adenin für den Organismus keineswegs als indifferent erwies, glaubte Minkowski, von Versuchen an Menschen von vornherein abstehen zu missen.

Nun ergaben die Untersuchungen, welche - zum grössten Theile im Laboratorium unserer Klinik - uber den Abbau der Purinkörper angestellt wurden, die bemerkenswerthe Thatsache, dass die einzelnen Purinkörper im Organismus verschiedener Thiergattungen in verschiedener Weise abgebaut werden, und es lag nahe, mit dieser Differenz auch die verschiedene Wirkungsweise dieser Körper bei den einzelnen Thieren in Beziehung zu bringen. So haben $\mathrm{K} \mathbf{r} \mathfrak{u} g \mathrm{er}$ und Sohmid ${ }^{1}$ ) für das Theobromin, Krüger ${ }^{2}$ ) fur das Coffein gezeigt, dass sie beim $\mathrm{Hunde}$ anders abgebaut werden, als beim Kaninchen. ${ }^{3}$ ) Die Uebereinstimmung der Art und Weise des Abbaues beim letzteren Thiere mit der im menschlichen Organismus legte nahe, bevor am Menschen mit Adenin Versuche gemacht werden konnten, an $\mathrm{K}$ aninchen einige Vorversuche anzustellen.

Ihr Ergebniss sei in Folgendem festgestellt:

\section{Verfutterung von Adenin am Kaninchen.}

Versuch I. Einem Kaninchen wurde mittelst Schlundsonde in Wasser gelöstes, freies Adenin eingegeben, und zwar erhielt es an 3 Tagen zweimal 0,25, an 3 Tagen einmal 0,5, und am letzten Tag dreimal 0,5 g, zusammen also 4,5 g Adenin. Am Tage nach Beendigung des Versuchs wurde es getödtet.

Versuch II. Das Kaninchen erhielt auf dieselbe Weise am 30 . und 31. Mai und 1. Juni zwei Mal 0,25, am 2., 3. und 4. Juni 0,5, am 5. und 6. Juni drei Mal 0,5, am 7. Juni Morgens, Mittags und Abends je 1,0, am 8. Juni drei Mal 1,5 $\mathrm{g}$, zusammen $13 \mathrm{~g}$ freies Adenin. Am 9. Juni wurde es getödtet.

Der Urin, welcher von beiden Kaninchen zusammen gesammelt wurde,

1) Ber. der deutschen chem. Gesellschaft, Bd. 32, S. 2677.

2) Ebenda, Bd. 32, S. 3336.

3) Vergl. auch die eben erschienene Mittheilung von Krüger und Sch mid über Entstehung der Harnsäure aus freien Purinbasen. Zeitschr. f. phys. Chem., Bd. XXXIV, Heft 5 u. 6, S. 560. 
enthielt schon am 2. Tage der Adeninfüterung zahlreiche Krystalle, welche in ihrer Form der des harnsauren Ammoniums ähnlich waren. Daneben fanden sich Tripelphosphate, Pseudocylinder mit Einlagerung obiger Krystalle und vereinzelte reine Pseudocylinder.

Das Sediment war sehr reichlich, die Farbe trttb gelblich-röthlich. Die Reaction war stark alkalisch. Eiweiss enthielt der Urin nicht. Der mikroskopische Befund blieb während des ganzen Versuches derselbe.

Der Sectionsbefund bot, abgesehen von den Veränderungen in der Niere, welche weiter unten beschrieben werden, nichts Besonderes, insbesondere keine Entzündungserscheinungen im Magen-Darmkanal.

Die chemische Untersuchung des gesammelten Harnes beider Thiere auf Harnsäure und Basen wurde nach der Methode von Kruger und Schmid ausgefuhrt: Es wurde 0,1 $\mathrm{g}$ Harnsäure erhalten, und aus dem Filtrat der Harnsäure konnte durch Fällung mit Pikrinsäure $12 \mathrm{~g}$ Adeninpikrat $=4,24 \mathrm{~g}$ reines Adenin gewonnen werden. Es hat also keine Vermehrung der Harnsäure stattgefunden. Dagegen sind 24,2 Proc. des eingegebenen Adenins im Harn wieder erschienen.

Aus diesen Versuchen ergab sich des Weiteren, in Uebereinstimmung mit unserer Vermuthung, dass Aden in thatsächlich beim $\mathrm{Ka}$ ninchen im Gegensatz zum Hunde eine andere Wirkung zeigt, indem es in ausserordentlich grossen Dosen, ohne erkennbarschädliche Wirkung ertragen wurde. Die Thiere befanden sich bis zum Schluss des Versuchs durehaus wohl und zeigten nicht einmal verminderte Fresslust.

\section{Veränderungen der Kaninohenniere nach Adenin- verfutterung.}

Bei dem ersten Kaninchen, welches nur kleine Dosen Adenin be. $\mathrm{kam}$, fanden sich keine Veränderungen in der Niere.

Um so auffallender war der Befund bei dem zweiten Kaninchen, welches $13 \mathrm{~g}$.Adenin erhielt. Die Oberfläche der in ihrer Grösse nicht veränderten $\mathrm{Ni}$ ie zeigte, nachdem die Kapsel liohne viele Mühe abgezogen war, zahllose feine, grauweisse Pünktehen. Auf dem Längsschnitt fanden sich eine Menge anscheinend den Nierenkanälchen entsprechender radiär verlaufender, grauweisser Striche und Streifen von verschiedener Ausdehnung, sowie mitunter grauweisse Punkte, analog denen an der Oberfäche. Diese Einlagerungen localisirten sich vornehmlich in der Rinde und in der Uebergangszone von Rinde zum Mark, während die Marksubstanz selbst beinahe ganz frei davon zu sein schien. Die Nierenbecken waren pathologisch nicht verändert.

Eine mikroskopische Untersuchung wurde zunächst am frischen Querschnitt vorgenommen. Man fand darin die Glomeruli vollkommen intact. In den Harnkanälchen dagegen traf man bald nach ihrem Austritt aus den Glomerulis bis hinab zur Grenze zwischen Rinde und Marksubstanz, entsprechend den makroskopisch sichtbaren Veränderungen, Einlagerung von theils reihenweise, theils einzelliegenden gelbbraunen Kugeln. Ihre Grösse war sehr verschieden; bald waren sie 5-6 Mal so gross, 
bald kleiner als die umgebenden Epithelien. Sie lagen, wenn gehäuft, meist hintereinander, manchmal auch zu einem Klumpen geballt. Meist zeigten sie nur in den Randpartien des Gebildes radiäre Streifung, während das Centrum aus einer dunklen Kugel bestand.

Die Niere wurde in Alkohol gehärtet, in Celloidin eingebettet, geschnitten und die Schnitte mit Hämatoxylin-Eosin gefärbt. Danach finden sich die rosettenartigen Gebilde stets innerhalb der Harnkanälchen liegend, welche je nach der Grösse und Menge der Einlagerungen mehr oder weniger dilatirt erschienen. Entzündungserscheinungen finden sich nirgends. Dagegen bewirken grössere Kugeln meist eine Abplattung der Epithelien, welche an dem sichtlich plattgedrückten Kern deutlich hervortrat. Manchmal schien es auch, als ob die Epithelzellen sich von der Wand abgehoben hätten und im Lumen der Harnkanälchen lägen. Jedoch fanden sich nirgends ausgesprochene Cylinderbildungen. Eine Abbildung der Rosetten an dieser Stelle scheint uberflussig, da sie mit den von Minkowski beschriebenen und sehr gut abgebildeten Sphärolithen ubereinstimmen.

Es sei noch das Resultat einiger, an ungefärbten Schnitten der in Alkohol gehärteten Niere vorgenommener mikroskopischer Reaction en mitgetheilt: Wasserzusatz verändert die kugeligen Gebilde nicht. Dagegen werden sie langsam gelöst durch Sodalösung, Ammoniak und Piperazinlösung, rasch durch Salzsäure und Natronlange. Auf keine Weise konnte eine Umkrystallisirung an Ort und Stelle bewirkt werden. Vielmehr verschwanden die Rosetten unter Hinterlassung von hellen Flecken, welche der Grösse und Lage derselben entsprechen.

Eine chemische Untersuchung der Krystalle aus zerhacktem Nierengew ebe gelang nicht, da auf diese Weise keine gentigende Ausbeute erzielt wurde. Trotzdem aber scheint es sowohl der Krystallform wie dem mikrochemischen Befunde nach, ausser Frage za stehen, dass es sich hier um Ablagerung von harnsaurem Ammoniak handelt.

Es reagirt also das Kaninchen in Bezug auf die pathologisch-anatomischen Veränderungen der Niere nach Adenineingabe in ähnlicher Weise, wie der Hund. Es bedarf jedoch dazu erheblich grösserer Mengen.

Ueber einen zum Vergleich angestellten Controlversuch am Hunde kann ich mich kurz fassen:

Das mittelgrosse Versuchsthier erhielt innerhalb 8 Tagen im Ganzen $7,4 \mathrm{~g}$ Adenin. Schon am zweiten Tage (nach Darreichung von 3-4 Mal $0,5)$ typischer Harnbefund:

Rosetten in der Krystallform des harnsauren Ammoniums, vereinzelte Uratcylinder, spärliche Nierenepithelien. In den nächsten Tagen kamen dazu zahlreiche weisse Blutkörperchen, Plattenepithelien, ganz vereinzelte hyaline Cylinder, kein Albumen. Eine quantitative Schätzung der Purinbasen im Harn ergab - vornehmlich im Vergleich mit dem Harn der oben erwähnten Kaninchen - nur einen auffallend geringen Niederschlag. Anatomisch ergab sich das von Minkowski ausfuhrlich geschilderte Bild, auf welches nicht näher eingegangen werden soll. Bemerkens- 
werth war dabei vor Allem die enorme Hyperämie sämmtlicher innerer Organe und zablreiche frische Blutungen im Herzmuskel, die an der Grenze zwischen Ventrikel und Vorhof am umfangreichsten waren. Keinerlei entzundliche Veränderungen. Endocard und Klappen frei. Dieser Befund deckt sich in gewissem Sinne mit den Angaben Minkowski's über das Verhalten des Circulationsapparates am lebenden Thiere nach Adeninzufuhr (sehr erregte Herzthätigkeit, auffallend verstärkter Herzstoss, vgl. insbesondere Versuch XX).

Die Verfütterung von Adenin hat ergeben, dass zwar keine Vermehrung von Harnsäure im Harne, wohl aber eine Ablagerung von Harnsäure und harnsaurem Ammonium in der Niere statthat. Da nun das Guanin, was die chemische Constitution betrifft, als Zwischenstufe beim Uebergange des Adenins in Harnsäure anzusehen ist; ergab sich aufs Neue die Frage: Wie verhält sich der thierische Organismus gegen Guanin? Versuche mit diesem Körper sind bereits früher schon wiederholt angestellt worden. Kerner ${ }^{1}$ ) hat an vier Tagen $25 \mathrm{~g}$ Guan in an $\mathrm{Kan}$ inchen verfuttert, ohne eine Zunahme der Harnsäure constatiren zu können. Da er sich aber der alten Methode von Heintz und Schwanert (Fällung der Harnsäure durch Salzsäure) bediente, so sind seine Versuche nicht maassgebend. Dann hat Stadthagen ${ }^{2}$ ) bei einem Hunde nach Eingabe von $6 g$ Guanin weder einen Anstieg der Harnsäure, noch der Basen constatiren können. Zum gleichen Resultat führten zwei neuere Versuche von $B u r i a n$ und $S c h u r^{3}$ ) am Menschen. Sie verfutterten einer Patientin an 3 Tagen 7,1 , einer zweiten $1,1 \mathrm{~g}$ Guanin. In letzter Zeit haben Krüger und Schmid diesen Versuch am Menschen wiederholt nnd dabei eine geringe, aber dentliche Vermehrung der Harnsäure, dagegen keine Vermehrung der Basen gefunden. Da ein einmaliger Versuch mit $0,6 \mathrm{~g}$ Guanin ihrerseits vorliegt, empfinden sie selbst das Bediurfniss einer Controlle. Endlich versprach - mit Rticksicht auf die oben geschilderten Befunde nach A d en in eingabe - auch hier die pathologisch-anatomische Untersuchung Interesse.

III. Verfutterung von Guanin am Kaninchen.

Versuch III. Einem Kaninchen wurde mittelst Schlundsonde in Wasser gelöstes Guaninsulfat einverleibt. Es erhielt am 1. Tage 0,3, am 2. zwei Mal 0,5 , am 3. Tage wurde ausgesetzt, am 4 . und 5 . Tage 0,5 , am 6 . und 7. Tage zwei Mal 0,5 g. Im Ganzen wurden also $5,3 \mathrm{~g}$ Guaninsulfat verftuttert.

1) Liebig's Annalen Nr. 103, S. 249.

2) Virchow's Archiv 109, S. 416.

3) Archiv für die gesammte Physiologie, Bd. 80. 
Eine mikroskopische Untersuchung des eiweissfreien Urins ergab in durch Centrifugiren gewonnenen Sediment als einzigen Befund spärliche Anzahl von Tripelphosphaten.

Das Kaninchen befand sich am Schlusse des Versuches, sowie die folgende Zeit ganz wohl. Als es nach einigen Wochen getödtet wurde, fanden sich bei der Section keine pathologischen Veränderungen, insbesondere keine Harnsäureablagerungen in der Niere.

Bei der chemischen Untersuchung des gesammelten Urins konnte, in Uebereinstimmung mit den früheren Autoren, keine Vermehrung der Harnsäure und der Basen festgestellt werden. 